\title{
NOMA-Based Irregular Repetition Slotted ALOHA for Satellite Networks
}

\author{
Xinye Shao, Student Member, IEEE, Zhili Sun, Senior Member, IEEE, Mingchuan Yang, Member, IEEE, Sai Gu, \\ and Qing Guo, Member, IEEE,
}

\begin{abstract}
In this letter, a non-orthogonal multiple access (NOMA) scheme is employed for irregular repetition slotted ALOHA (IRSA). Specifically, packet replicas are transmitted with discrete power levels which are pre-determined by the NOMA scheme. In this case, most packet collisions can be resolved in the power domain, contributing to a much lower packet loss rate. Density evolution (DE) analysis is formulated and the degree distributions are optimized for different number of power levels. Simulation results validate our analysis and show that the proposed scheme can outperform existing IRSA schemes.
\end{abstract}

Index Terms-Irregular repetition slotted ALOHA, nonorthogonal multiple access, interference cancellation, diversity, satellite communication.

\section{INTRODUCTION}

A $S$ the key enabler of Internet of Things (IoT), Machine to Machine (M2M) communications have received a lot of attention. M2M traffic is characterized by large number of terminals and bursty small packets, which makes the traditional Demand Assignment Multiple Access (DAMA) satellite protocol very inefficient due to the unacceptable signaling overhead and large delay.

The uncoordinated nature of random access (RA) well matches this type of traffic. However, packet collisions have become the main limitation. Channel sensing adopted in terrestrial networks to improve RA performance is not efficient in satellite environment [1]. Contention resolution slotted ALOHA (CRDSA) proposed in [2] first shed light on how to resolve packet collisions. The basic idea is to combine packet diversity and successive interference cancellation (SIC). Another great contribution was made in [3] where IRSA was introduced. In IRSA, the number of replicas is selected according to a probability mass function (PMF). By borrowing tools from the coding field, the optimal PMF is obtained.

The collision channel model has been widely used for designing IRSA schemes, where packets in a collision are lost and packets without a collision are successfully decoded. However, it was shown in [4] that packets involved in a collision can still be decoded due to the capture effect (CE). In [5], IRSA over Rayleigh block fading channel with $\mathrm{CE}$

This work was funded by National Natural Science Foundation of China, grant\#91438205 and \#91538104.

X. Shao, M. Yang and Q. Guo are with School of Electronics and Information Engineering, Harbin Institute of Technology, Harbin, P.R.China. (e-mail: shaoxinye,mcyang,qguo@hit.edu.cn)

Z. Sun is with Institute of Communications Systems, University of Surrey, Guildford GU2 7XH, U.K. (e-mail: z.sun@surrey.ac.uk)

S. Gu is with Department of Chemical and Process, University of Surrey, Guildford GU2 7XH, U.K. (e-mail: sai.gu@ surrey.ac.uk) was analyzed and optimized. The authors in [6] obtained the optimal received packet power distribution for CRDSA with CE. Discrete power levels and inter-slot power diversity were introduced in [7] to improve CRDSA, where the optimal distribution of power levels was derived. However, the way to determine the power levels was not given.

In non-orthogonal multiple access (NOMA), the same resource is shared by multiple users. The receiver utilizes power difference and SIC to decode user signals. Generally, coordinations with known channel state information (CSI) are needed to allocate powers to different users. For this reason, NOMA seems not suitable for RA schemes which are lack of coordinations. However the author in [8] demonstrated that RA can also benefit from NOMA. This has motivated us to investigate the application of NOMA in IRSA. To the best of our knowledge, no NOMA scheme has been investigated for IRSA.

In this letter, in addition to packet diversity, power diversity is utilized through sending packet replicas with different power levels. The novelty is that our scheme leverages on a NOMA scheme to specify the discrete power levels and we investigate it for IRSA. We analyze the performance of the proposed scheme in the asymptotic regime by deriving the DE recursion functions under different number of power levels. Based on this, we then maximize the scheme throughput through differential evolution [9]. We show that our scheme can improve the packet loss rate (PLR) performance w.r.t the existing IRSA schemes.

The letter is organized as follows. Section II introduces the system model. The proposed NOMA-Based IRSA scheme is described in Section III. Section IV presents the asymptotic performance analysis of the proposed scheme considering different number of power levels. Simulation results are provided in Section V. Section VI concludes the letter.

\section{SYSTEM MODEL}

We consider a scenario where $M$ M2M users send packets over a shared medium on the uplink of a satellite. $M$ is a random variable with poisson distribution. After registering in the system, each user will keep TDMA slot synchronization. All users transmit their packets on a frame basis and the packet length is equal to the slot length. Each frame consists of $N$ slots. The system load $G$ is defined as $\frac{M}{N}$ [packet/slot]. Packets will be transmitted only once, i.e., retransmission is not considered.

In IRSA, a user transmits $l$ replicas of its packet in a frame according to a PMF: $\left\{\Lambda_{l}\right\}$, where $\Lambda_{l}$ represents the probability 


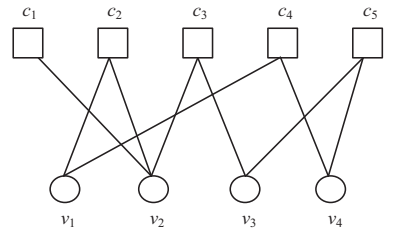

Fig. 1. An example of bipartite representation of IRSA with $N=5$ and $M=4$.

that a user transmits $l$ replicas. The positions of the replicas are uniformly distributed within the frame. Each replica will contain in its head the positions of other replicas. If one replica is successfully decoded, the rest replicas are reconstructed and subtracted from the frame, which cancels the interference induced by those replicas, thus allowing more packets to be decoded.

IRSA can be described by a bipartite graph. An example with $M=4$ and $N=5$ is illustrated in Fig. 1. User $i$ is represented by a variable node $(\mathrm{VN}) v_{i}$ denoted by a circle and slot $j$ represented by a check node $(\mathrm{CN}) c_{j}$ denoted by a square. $v_{i}$ is connected to $c_{j}$ if and only if a replica of user $i$ is transmitted in slot $j$. The degree of $v_{i}$ is defined as the number of replicas transmitted by user $i$ and the degree of $c_{j}$ defined as the number of packets transmitted in slot $j$. Thus, $c_{j}$ is collision-free when its degree is 1 . Based on this, from Fig. 1, we can know that only $c_{1}$ is collision-free. Under the collision channel model, the replica of user 2 in slot 1 is first decoded.

\section{NOMA-BASED IRSA SCHEME}

\section{A. Description of the NOMA Scheme}

A NOMA scheme in [10] is considered. For a specific user, let $h$ denote the channel coefficient representing the ratio between received and transmitted signal power for each packet. $h$ can be estimated using the pilot signal broadcasted by the satellite. Perfect channel estimation is assumed for simplicity. Suppose that there are $L$ packet power levels at the satellite receiver side denoted by

$$
p r_{1}>\ldots>p r_{L}>0
$$

where $p r_{k}$ represents the power of level $k$. The user can randomly choose any power level, for instance $p r_{k}$, for access. In this case, the user transmission power $p t$ is calculated by $p t=\frac{p r_{k}}{h}$. Normalizing the spectral density of the background noise, the signal-to-interference-plus-noise ratio (SINR) of the user at the satellite is simply $p r_{k}$. The power levels in (1) are specified by

$$
p r_{k}=\Gamma\left(P r_{k}+1\right),
$$

where $\Gamma$ is the target SINR and $P r_{k}=\sum_{i=k+1}^{L} p r_{i}$ with $\operatorname{Pr}_{L}=0$. The value of $\Gamma$ is associated with the desired transmission rate $R$ through Shannon formula

$$
R=\log (1+\Gamma) .
$$

We can get $p r_{L}=\Gamma$ by letting $k=L$ in equation (2). Substituting $p r_{L}$ in equation (2) produces $p r_{L-1}=\Gamma(\Gamma+1)$. Continuing this process, it can be shown that

$$
p r_{k}=\Gamma(\Gamma+1)^{L-k} .
$$

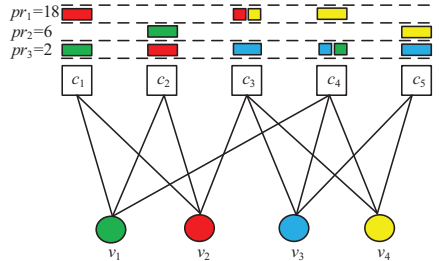

Fig. 2. An example of bipartite representation of NOMA-Based IRSA with $L=3, N=5, \Gamma=3 \mathrm{~dB}$ and $M=4$.

Suppose that there exist $L$ active users, each choosing a different power level. Then the SINR of the user with power level $p r_{1}$ will be $\frac{p r_{1}}{P r_{1}+1}$, which is $\Gamma$ from (2). This user can be decoded and cancelled by SIC. Then the SINR of the user with power level $p r_{2}$ becomes $\Gamma$, being able to be decoded and removed. As a result, all active users can be decoded in ascending order of their power level although they are transmitting on the same channel. It can be easily shown that when the number of active users is less than $L$, all users can also be decoded.

\section{B. IRSA Based on NOMA}

Assume the transmission rate is $R$. Then the target SINR $\Gamma$ is calculated from (3) as $\Gamma=2^{R}-1$. For the user performing IRSA, a power level is chosen independently and uniformly from the pre-determined power levels for each packet replica for transmission. Based on (1), the maximal power is $p r_{1}$. From (4), it can be seen that $p r_{1}=\Gamma(\Gamma+1)^{L-1}$ increases exponentially with $L-1$. In practice, $L$ should not be too large considering the limited energy of M2M terminals.

Due to the lack of coordinations, different users may choose the same power level, leading to power collisions. Assuming two packets collide at level $k$, the SINR of the packets is given by $B=\frac{p r_{k}}{p r_{k}+1}$. Since $p r_{k}>0$, it is obvious that $B<1$. In practice, it is reasonable to assume $\Gamma>0 \mathrm{~dB}$, i.e., $\Gamma>$ 1, which means $B<\Gamma$. Therefore, the colliding packets at level $k$ can not be decoded. Due to the SIC process of the receiver, the decoding of packets at different power levels is not independent. The SIC process will get stuck at level $k$. As a result, all the packets at levels $k+1, \ldots, L$ will not be decoded even when there are no collisions at these levels. However, a packet at higher levels $1, \ldots, k-1$ might be able to be decoded if the packet SINR $B$ meets the condition $B \geq \Gamma$. This is depicted by an example in Fig. 2, where $L=3, M=4$, $\Gamma=3 \mathrm{~dB}$ and $N=5$.

In this graph, we associate different users with different colors. Above each $\mathrm{CN}$, a three-row table is used to represent the three power levels. In addition to the lines between CNs and VNs, packets are further identified with colors and power levels. Thanks to the NOMA scheme introduced, both packets in $c_{1}$ can be decoded since they are at different power levels. In $c_{3}$, the packet at lower level 3 can not be decoded due to the strong interference from colliding packets at level 1. While in $c_{4}$, the packet at higher level 1 can be decoded for the SINR $B=\frac{18}{2+2+1}=3.6>\Gamma=2$, although there is a collision at lower level 3 . 


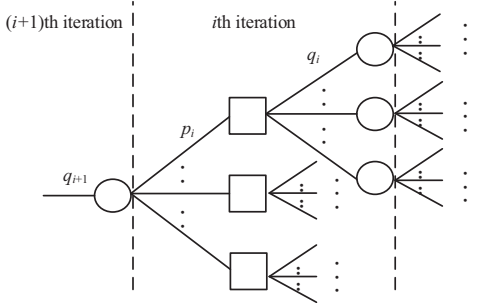

Fig. 3. Tree representation in the asymptotic regime.

\section{DENSITY EVOLUTION ANALYSIS}

Suppose the average traffic load is $G$ [packet/slot]. It has been shown in [3] that the degree distribution of VNs from node and edge perspective can be expressed in polynomial forms as

$$
\Lambda(x)=\sum_{l} \Lambda_{l} x^{l}, \quad \lambda(x)=\sum_{l} \lambda_{l} x^{l-1},
$$

where $\lambda_{l}$ denotes the probability that an edge is connected to a degree-l $\mathrm{VN}$ and can be computed as $\lambda_{l}=\Lambda_{l} l / \sum_{k} \Lambda_{k} k$. Similarly to VNs, $\rho_{l}$ represents the probability that an edge is connected to a degree- $l \mathrm{CN}$ and is calculated as $\rho_{l}=$ $\Psi_{l} l / \sum_{m} \Psi_{m} m$, where $\Psi_{l}=\frac{\left(G \Lambda^{\prime}(1)\right)^{l}}{l !} e^{-G \Lambda^{\prime}(1)}$ is the probability that a $\mathrm{CN}$ has degree $l$; here $\Lambda^{\prime}(x)=d \Lambda(x) / d x$, so $\Lambda^{\prime}(1)=\sum_{l} \Lambda_{l} l$.

In the asymptotic regime, i.e., $N \rightarrow \infty$, there is no loop in the bipartite graph. In this case, a tree can be obtained by unfolding the graph from a randomly chosen edge as depicted in Fig. 3. In the proposed scheme, a packet is able to be erased from the frame when it can be decoded directly or any of its replicas can be decoded. Denote by $q_{i}$ the probability that a packet can not be erased through its replicas in the $i$ th iteration and $p_{i}$ the probability that a packet can not be decoded directly in the $i$ th iteration. Let $P(r, L, \Gamma)$ denote the probability that a packet can be decoded directly when the number of power level is $L$, the target SINR is $\Gamma$ and there exist other $r-1$ interfering packets in the same slot. Based on this, we can get

$$
\begin{gathered}
q_{i+1}=\sum_{l} \lambda_{l} p_{i}^{l-1}=\lambda\left(p_{i}\right), \\
p_{i}=1-\sum_{l=1}^{\infty} \rho_{l} \sum_{r=1}^{l} P(r, L, \Gamma)\left(\begin{array}{l}
l-1 \\
r-1
\end{array}\right) q_{i}^{r-1}\left(1-q_{i}\right)^{l-r} .
\end{gathered}
$$

By changing the order of summation, (7) can be further simplified as

$$
\begin{aligned}
p_{i} & =1-\sum_{r=1}^{\infty} \sum_{l=r}^{\infty} \rho_{l} P(r, L, \Gamma)\left(\begin{array}{c}
l-1 \\
r-1
\end{array}\right) q_{i}^{r-1}\left(1-q_{i}\right)^{l-r} \\
& =1-e^{-q_{i} G \Lambda^{\prime}(1)} \cdot \sum_{r=1}^{\infty} \frac{\left(q_{i} G \Lambda^{\prime}(1)\right)^{r-1}}{(r-1) !} P(r, L, \Gamma) .
\end{aligned}
$$

As mentioned earlier, whether a packet can be decoded depends on the specific collision pattern and $\Gamma$. As there are $L^{r}$ different collision patterns when $r$ packets are transmitted in the same slot at $L$ power levels, it is difficult to obtain an analytical expression for $P(r, L, \Gamma)$. Recall that when $r \leq L$ and every packet is transmitted at a different power level, all $r$ packets can be decoded. Hence by only considering this situation, a lower bound $\underline{P}(r, L)$ for $P(r, L, \Gamma)$ can be derived as

$$
\underline{P}(r, L)=\prod_{i=1}^{r-1}\left(1-\frac{i}{L}\right) .
$$

Note that $\underline{P}(r, L)=0$ when $r>L$. Therefore, (9) also applies to cases with $r>L$. When $L \leq 2$, packets can be successfully decoded only when they are transmitted at different power levels. In this case, the lower bound becomes exact. In the following, we use this lower bound as an approximation to $P(r, L, \Gamma)$. By replacing $P(r, L, \Gamma)$ in (8) with (9), an approximation to $p_{i}$ is obtained as

$$
p_{i} \approx 1-e^{-q_{i} G \Lambda^{\prime}(1)}(L-1) ! \sum_{r=1}^{L} \frac{\left(q_{i} G \Lambda^{\prime}(1) / L\right)^{r-1}}{(r-1) !(L-r) !} .
$$

Combing (10) and (6), the DE recursion for $p_{i}$ can be approximated as

$$
\begin{aligned}
p_{i+1} & \approx 1-e^{-\lambda\left(p_{i}\right) G \Lambda^{\prime}(1)}(L-1) ! \sum_{r=1}^{L} \frac{\left(\lambda\left(p_{i}\right) G \Lambda^{\prime}(1) / L\right)^{r-1}}{(r-1) !(L-r) !} \\
& =f\left(p_{i}, G, L,\left\{\Lambda_{l}\right\}\right) .
\end{aligned}
$$

As there are no packets recovered in the beginning of the SIC process, $q_{0}$ is set to be 1 . Then $p_{0}$ can be calculated by using (10). Applying $p_{0}$ to (11), a DE limit $p_{\infty}\left(G, L,\left\{\Lambda_{l}\right\}\right)=$ $\lim _{i \rightarrow \infty} p_{i}$ can be achieved. Since a packet has $l$ replicas with probability $\Lambda_{l}$ and a packet is considered to be lost when all its replicas can not be decoded, the PLR in the asymptotic regime is given by $\operatorname{PLR}\left(G, L,\left\{\Lambda_{l}\right\}\right)=\sum_{l} \Lambda_{l} p_{\infty}\left(G, L,\left\{\Lambda_{l}\right\}\right)^{l}$.

In asymptotic regime, packets are expected to be recovered with a probability close to 1 when $G$ is below a specific threshold $G^{*}$ and will be lost with a probability bounded away from 0 [3]. With the DE recursion derived, we can define $G^{*}$ as the maximal value of $G$ such that $f\left(p, G, L,\left\{\Lambda_{l}\right\}\right)<p, \forall p \epsilon(0,1]$. Our purpose is to optimize $\left\{\Lambda_{l}\right\}$, i.e., $\Lambda(x)$ through differential evolution to maximize $G^{*}$.

\section{NUMERICAL RESULTS}

The maximal repetition rate and $\Gamma$ is assumed to be 8 and $3 \mathrm{~dB}$ respectively. For an exact DE recursion, we combine (8) and (6). Denote by $\Lambda_{L}(x)$ the optimal $\Lambda(x)$ with $L$ power levels. $\Lambda_{L}(x)$ optimized through the exact and approximated DE with different $L$ are given in Table I. Note that when $L \leq$ 2 , the approximated method becomes exact. The asymptotic performance, i.e., $G^{*}$ of different $\Lambda_{L}(x)$ are compared in Fig. 4. It can be seen that a good approximation can be achieved by the approximated DE derived. On the other hand, $G^{*}$ increases linearly with the number of power levels, which translates to an improvement in throughput. In the following, $\Lambda_{L}(x)$ obtained by the approximated DE are used for simulation. The theoretical asymptotic PLR performance and the simulation results with finite frame sizes are compared in Fig. 5. It can be seen that the asymptotic PLR curve shows a threshold phenomenon as stated earlier. As we increase the frame length the PLR curve becomes steeper and approaches the threshold predicted by the theoretical threshold, validating our analysis. 


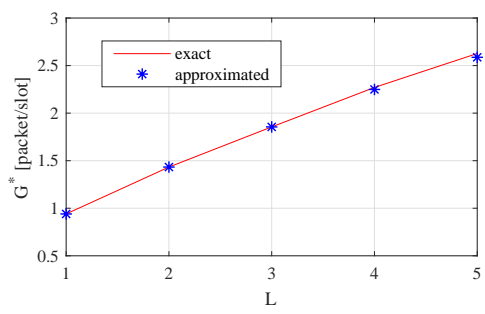

Fig. 4. Comparison of asymptotic performance of $\Lambda_{L}(x)$ optimized by exact and approximated density evolution with different $L$ and $\Gamma=3 \mathrm{~dB}$.

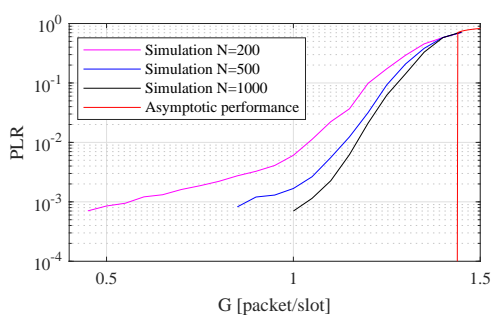

Fig. 5. Comparison of theoretical asymptotic performance and simulation results with finite frame sizes, 20 iterations, $\Lambda_{2}(x)$.

For comparison, we consider a recent work in [6], where the received packet power is distributed in a continuous range $\left[p_{\min }, p_{\max }\right]$. It was found that the optimal power distribution can be approximated by a uniform distribution. Differently, in our scheme, discrete power levels are specified by a NOMA scheme according to the transmission rate $R$. The power distribution is not subject to optimization. For the two schemes, the same average received packet power is assumed. $p_{\min }$ is set to be $\Gamma$ and $p_{\max }$ is calculated as $2 \frac{\sum_{k=1}^{L} p r_{k}}{L}-\Gamma$. Simulation results of PLR performance for the two schemes are provided in Fig. 6 with $N=200$. The scheme in [6] and our scheme are denoted by IRSA-C and IRSA-NOMA respectively.

For IRSA-NOMA, targeting a $P L R=10^{-3}, \Lambda_{1}(x)$ would be able to operate at $G \simeq 0.27$. While $\Lambda_{2}(x)$ can reach up to $G \simeq 0.56$ and $\Lambda_{3}(x)$ allows achieving that loss rate at $G \simeq 0.87$. This indicates the benefit brought by larger number of power levels. On the other hand, IRSA-C operates at relatively low loads, $G \simeq 0.17$ and $G \simeq 0.23$ for $\Lambda_{2}(x)$ and $\Lambda_{3}(x)$ respectively. This can be explained that colliding packets can be decoded using SIC if the power differences are sufficiently large. In IRSA-NOMA, discrete power levels and the power differences are carefully designed by a NOMA scheme. While in the uniform continuous power case, this consideration is missing, so packets are received with closer power with a relatively higher probability, leading to a lower throughput. Note that the threshold-based decoding model adopted in our analysis assumes that capacity-achieving codes are used and perfect interference cancellation is performed, which will have some effect on the performance of the scheme. Nevertheless, our results can be used as a first approximation for highlighting the improvements given by the proposed scheme.
TABLE I

$\Lambda_{L}(x)$ OBTAINED BY THE EXACT AND APPROXIMATED DE.

\begin{tabular}{ccc}
\hline$L$ & DE & $\Lambda_{L}(x)$ \\
\hline 1 & exact & $0.5112 x^{2}+0.266 x^{3}+0.2228 x^{8}$ \\
2 & exact & $0.6607 x^{2}+0.1605 x^{3}+0.1788 x^{8}$ \\
3 & exact & $0.7439 x^{2}+0.0906 x^{3}+0.0156 x^{4}+0.1499 x^{8}$ \\
& approximated & $0.7606 x^{2}+0.055 x^{3}+0.0224 x^{4}+0.162 x^{8}$ \\
4 & exact & $0.7947 x^{2}+0.047 x^{3}+0.1583 x^{8}$ \\
& approximated & $0.809 x^{2}+0.0342 x^{3}+0.1568 x^{8}$ \\
5 & exact & $0.837 x^{2}+0.163 x^{8}$ \\
& approximated & $0.8499 x^{2}+0.1501 x^{8}$ \\
\hline
\end{tabular}

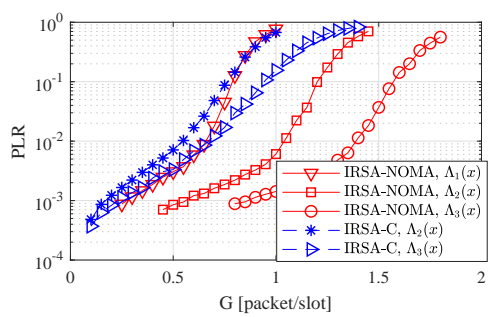

Fig. 6. Simulated PLR performance of IRSA-C and IRSA-NOMA for $\Lambda_{L}(x)$ with $N=200, L=1,2,3.20$ iterations.

\section{CONCLUSION}

In this letter, we proposed a NOMA-Based IRSA scheme utilizing the power dimension provided by a NOMA scheme. We formulated DE analysis for the new scheme and optimized the scheme throughput by differential evolution under different number of power levels. It is shown that the proposed scheme is able to outperform the existing IRSA schemes. Simulation results in finite frame length regime validate our analysis.

\section{REFERENCES}

[1] R. D. Gaudenzi and O. del Rio Herrero, "Advances in random access protocols for satellite networks," in Proc. 2009 International Workshop Satellite and Space Commun., Tuscany, Italy, Sep. 2009, pp. 331-336.

[2] E. Casini, R. D. Gaudenzi, and O. del Rio Herrero, "Contention resolution diversity slotted ALOHA (CRDSA): An enhanced random access scheme for satellite access packet networks," IEEE Trans. Wireless Commun., vol. 6, no. 4, pp. 1408-1419, Apr. 2007.

[3] G. Liva, "Graph-based analysis and optimization of contention resolution diversity slotted ALOHA," IEEE Trans. Commun., vol. 59, no. 2, pp. 477-487, Feb. 2011.

[4] A. Mengali, R. D. Gaudenzi, and C. Stefanovic, "On the modeling and performance assessment of random access with SIC," IEEE J. Sel. Areas Commun., vol. 36, no. 2, pp. 292-303, Feb. 2018.

[5] F. Clazzer, E. Paolini, I. Mambelli, and C. Stefanovic, "Irregular repetition slotted ALOHA over the Rayleigh block fading channel with capture," in Proc. IEEE Int. Conf. Commun., Paris, France, May. 2017, pp. $1-6$.

[6] A. Mengali, R. D. Gaudenzi, and P. Arapoglou, "Enhancing the physical layer of contention resolution diversity slotted ALOHA," IEEE Trans. Commun., vol. 65, no. 10, pp. 4295-4308, Oct. 2017.

[7] S. Alvi, S. Durrani, and X. Zhou, "Enhancing CRDSA with transmit power diversity for machine-type communication," IEEE Trans. Veh. Technol., vol. 67, no. 8, pp. 7790-7794, Aug. 2018.

[8] J. Choi, "NOMA-based random access with multichannel ALOHA," IEEE J. Sel. Areas Commun., vol. 35, no. 12, pp. 2736-2743, Dec. 2017.

[9] S. Rainer and P. Kenneth, "Differential evolution - a simple and efficient heuristic for global optimization over continuous spaces," J. Global Optimization, vol. 11, no. 4, pp. 341-359, Dec. 1997.

[10] J. Choi, "Re-transmission diversity multiple access based on SIC and HARQ-IR," IEEE Trans. Commun., vol. 64, no. 11, pp. 4695-4705, Nov. 2016. 